\title{
Results of spinal accessory to suprascapular nerve transfer in 110 patients with complete palsy of the brachial plexus
}

\author{
Jayme Augusto Bertelli, MD, PhD, ${ }^{1,2}$ and Marcos Flávio Ghizoni, MD, MSc ${ }^{1}$
}

'Department of Neurosurgery, Southern University of Santa Catarina (Unisul), Tubarão; and 'Department of Orthopedic Surgery, Governador Celso Ramos Hospital, Florianópolis, Santa Catarina, Brazil

\begin{abstract}
OBJECTIVE Transfer of the spinal accessory nerve to the suprascapular nerve is a common procedure, performed to reestablish shoulder motion in patients with total brachial plexus palsy. However, the results of this procedure remain largely unknown.

METHODS Over an 11-year period (2002-2012), 257 patients with total brachial plexus palsy were operated upon in the authors' department by a single surgeon and had the spinal accessory nerve transferred to the suprascapular nerve. Among these, 110 had adequate follow-up and were included in this study. Their average age was 26 years (SD 8.4 years), and the mean interval between their injury and surgery was 5.2 months (SD 2.4 months). Prior to 2005, the suprascapular and spinal accessory nerves were dissected through a classic supraclavicular L-shape incision $(n=29)$. Afterward $(n=81)$, the spinal accessory and suprascapular nerves were dissected via an oblique incision, extending from the point at which the plexus crossed the clavicle to the anterior border of the trapezius muscle. In 17 of these patients, because of clavicle fractures or dislocation, scapular fractures or retroclavicular scarring, the incision was extended by detaching the trapezius from the clavicle to expose the suprascapular nerve at the suprascapular fossa. In all patients, the brachial plexus was explored and elbow flexion reconstructed by root grafting $(n=95)$, root grafting and phrenic nerve transfer $(n=6)$, phrenic nerve transfer $(n=1)$, or third, fourth, and fifth intercostal nerve transfer. Postoperatively, patients were followed for an average of 40 months (SD 13.7 months).
\end{abstract}

RESULTS Failed recovery, meaning less than $30^{\circ}$ abduction, was observed in $10(9 \%)$ of the 110 patients. The failure rate was $25 \%$ between 2002 and 2004, but dropped to $5 \%$ after the staged/extended approach was introduced. The mean overall range of abduction recovery was $58.5^{\circ}\left(\mathrm{SD} 26^{\circ}\right)$. Comparing before and after distal suprascapular nerve exploration (2005-2012), the range of abduction recovery was $45^{\circ}\left(\operatorname{SD~} 25.1^{\circ}\right)$ versus $62^{\circ}\left(\operatorname{SD} 25.3^{\circ}\right)$, respectively $(p=0.002)$. In patients who recovered at least $30^{\circ}$ of abduction, recovery of elbow flexion to at least an $\mathrm{M} 3$ level of strength increased the range of abduction by an average of $13^{\circ}(p=0.01)$. Before the extended approach, $2(7 \%)$ of 29 patients recovered active external rotation of $20^{\circ}$ and $120^{\circ}$. With the staged/extended approach, 32 (40\%) of 81 recovered some degree of active external rotation. In these patients, the average range of motion measured from the thorax was $87^{\circ}\left(\mathrm{SD} 40.6^{\circ}\right)$.

CONCLUSIONS In total palsies of the brachial plexus, using the spinal accessory nerve for transfer to the suprascapular nerve is reliable and provides some recovery of abduction for a large majority of patients. In a few patients, a more extensive approach to access the suprascapular nerve, including, if necessary, dissection in the suprascapular fossa, may enhance outcomes.

http://thejns.org/doi/abs/10.3171/2015.8.SPINE15434

KEY WORDS spinal accessory nerve; brachial plexus; nerve transfer; nerve grafting; suprascapular nerve; technique; peripheral nerve

$\mathrm{S}$ URGICAL reconstruction of the shoulder is a high priority in patients with total brachial plexus injuries. This is not only because functional control of the shoulder is of paramount importance, but also because of the overall reduced success of reinnervation of muscles below the elbow.? In total lesions of the brachial plexus, the most common approach to reconstructing shoulder function is to transfer the spinal accessory nerve to the suprascapular nerve. ${ }^{14}$ The spinal accessory nerve may be concomitantly injured following stretch lesions of the bra- 
chial plexus, but it is preserved in almost $95 \%$ of the patients with brachial plexus palsy. ${ }^{4}$ Despite the widespread use of this nerve transfer procedure, the results are largely unknown, with some surgeons indicating disappointing results. ${ }^{12}$ Based on less-than-ideal results obtained after accessory to suprascapular nerve transfers, a few surgeons have proposed sparing the spinal accessory nerve, thereby preserving trapezius function with the ultimate goal of performing shoulder arthrodesis. ${ }^{1}$

In the present paper, we reviewed our 11-year experience performing spinal accessory nerve to suprascapular nerve transfers in 110 patients with complete palsy of the brachial plexus and prolonged follow-up. To the best of our knowledge, this is the largest published series of such nerve transfers for total palsy. Over the duration of the study, the surgical technique underwent modifications related to exposing either the suprascapular nerve or the spinal accessory nerve, and the influence of these modifications on our results is documented.

\section{Methods \\ Patients}

Over an 11-year period (2002-2012), 561 patients with brachial plexus palsy were operated upon in our department, all by a single surgeon. Among them, there were 257 patients with complete palsy following a closed supraclavicular injury affecting the brachial plexus. Patients who had surgery to transfer the spinal accessory nerve to the suprascapular nerve within the 1st year after their injury and had at least 2 years' follow-up were included in the present analysis. Patients with passive limitations of shoulder joint motion $(n=5)$, patients who had surgery more than 12 months after their injury $(n=7)$, patients with a weak trapezius $(\mathrm{n}=12)$, and patients with a short duration of follow-up $(\mathrm{n}=123)$ were excluded. Ultimately, a total of 110 patients met our criteria for inclusion, and they constitute the group for the present study. The patients' mean age at the time of surgery was 26 years (SD 8.4 years), and the mean time between the injury and surgery was 5.2 months (SD 2.4 months). Before surgery, all patients underwent a $\mathrm{CT}$ tomomyeloscan. The clinical and demographic characteristics of the patients are summarized in Table 1.

All patients underwent surgical repair in the supine position under general anesthesia. We explored the brachial plexus either using a classic L-shaped incision $(n=$ 29) or via another oblique incision that was made slightly lateral to Chassaignac's tubercle $(\mathrm{n}=81)$, as per previous reports. ${ }^{6}$ In 95 patients, a viable upper root was identified by microscopic inspection, and this was grafted to restore elbow flexion either to the anterior division of the upper trunk, or to the musculocutaneous nerve. In 6 patients, we judged the root to be moderately compromised and combined root grafting and phrenic nerve transfer to the musculocutaneous nerve using sural grafts. In 9 patients, all roots were avulsed. In 8 of these patients, the third, fourth, and fifth intercostal nerves were transferred directly to the musculocutaneous nerve, while in 1 patient with rib fractures and chest drainage we grafted the phrenic nerve to the musculocutaneous nerve using a sural graft.

In the first 29 patients, all operated upon between 2002
TABLE 1. Summary of patient clinical and demographic characteristics stratified by surgical approach*

\begin{tabular}{|c|c|c|}
\hline Variable & $\begin{array}{c}\text { Classic } \\
\text { Approach }\end{array}$ & $\begin{array}{c}\text { Staged/Extended } \\
\text { Approach }\end{array}$ \\
\hline No. of patients & 29 & 81 \\
\hline Mean age, yrs & 25.9 (SD 10.2) & 25.7 (SD 7.7) \\
\hline $\begin{array}{l}\text { Mean no. of months btwn injury } \\
\quad \& \text { op }\end{array}$ & 4.8 (SD 2.2) & $5.2(\mathrm{SD} 2.3)$ \\
\hline Mean follow-up in months & 39.9 (SD 11.4) & 39.8 (SD 14.2) \\
\hline \multicolumn{3}{|l|}{ Recovery } \\
\hline Shoulder abduction & $45^{\circ}\left(\operatorname{SD} 25.1^{\circ}\right)$ & $62^{\circ}\left(\operatorname{SD} 25.3^{\circ}\right) \dagger$ \\
\hline \multicolumn{3}{|l|}{ External rotation } \\
\hline No. of patients $\ddagger$ & 2 & $32 \dagger$ \\
\hline Range of motion & $20^{\circ}, 120^{\circ}$ & $87^{\circ}\left(\mathrm{SD} 40.6^{\circ}\right)$ \\
\hline Failure rate & $25 \% \S$ & $5 \%$ \\
\hline
\end{tabular}

* There were statistically significant differences in the recovery of shoulder abduction $(p=0.002)$ and in the number of patients who recovered some degree of external rotation $(p=0.009)$. The number of surgical failures, i.e., cases in which patients recovered less than $30^{\circ}$ of abduction, was significantly higher in the group treated with the classical approach group $(p=0.01)$. $\dagger$ Statistically significant.

$\ddagger$ Number of patients who recovered any degree of external rotation. $\S$ Mean value significantly greater than for classic approach $(p=0.01)$.

and 2004, inclusive, the spinal accessory to suprascapular nerve transfer was performed through a classic L-shaped incision in the supraclavicular region (i.e., classic approach). In the remaining 81 patients, who all underwent repair from 2005 onward, the suprascapular nerve was dissected through an oblique incision over the trapezius muscle (staged/extended approach) (Fig. 1). After the deep cervical fascia was detached from the trapezius, the spinal accessory nerve was readily identified. In 17 of these latter 81 patients, to properly locate a healthy stump of the suprascapular nerve, the skin incision was prolonged, in a zigzag fashion, over the trapezius up to the acromion. The trapezius muscle was then detached from the clavicle, and, after nerve repair, the trapezius was reinserted. In 7 of these patients, because of retroclavicular scarring due to a clavicular fracture $(\mathrm{n}=2)$, acromioclavicular dislocation $(n=1)$, sternoclavicular dislocation $(n=1)$, scapular fracture $(\mathrm{n}=1)$, rupture of the suprascapular nerve at the notch $(\mathrm{n}=1)$, or an extended lesion of the suprascapular nerve $(\mathrm{n}=1)$, a healthy suprascapular nerve stump was only identified after suprascapular fossa exploration. In 2 patients with a clavicular fracture and malunion, the distal third of the clavicle was osteotomized to improve access to the suprascapular notch. After suprascapular nerve repair, a tension band ensured clavicle stabilization. Details of the surgical technique to transfer the spinal accessory nerve to the suprascapular nerve are reported elsewhere. ${ }^{5,8}$ Short nerve grafts, approximately $2 \mathrm{~cm}$ long, were needed in only 3 of the 29 patients operated upon via the classic approach. In none of the patients operated by the staged/ extended approach was a nerve graft needed.

\section{Postoperative Care and Evaluation}

In the postoperative period, only a sling was used, and 


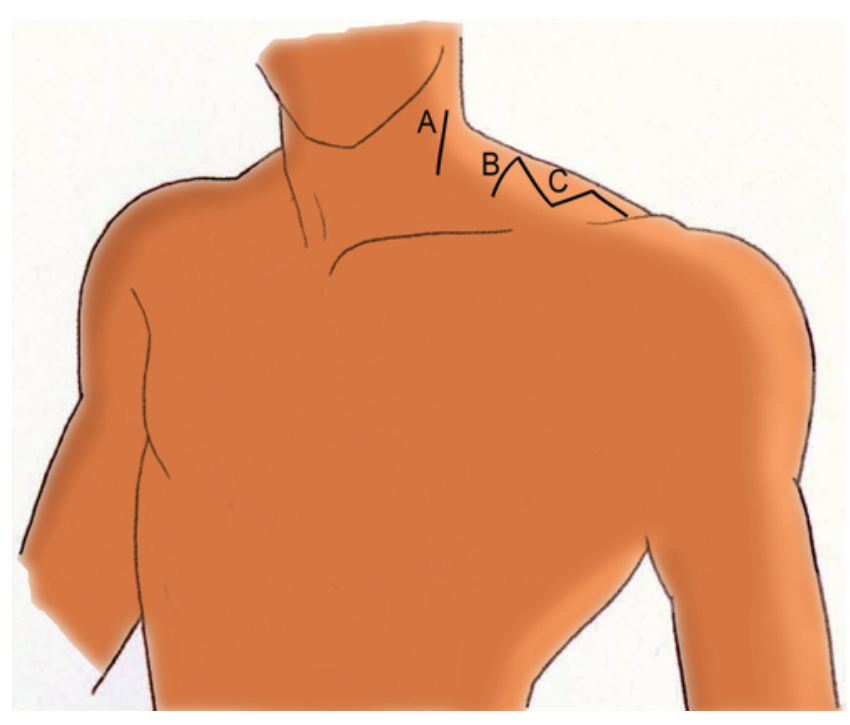

FIG. 1. Schematic representation of the surgical incisions used to explore the upper roots of the brachial plexus $(A)$ and to transfer the accessory to the suprascapular nerve in the limited approach (B) and extended approach (C). Figure is available in color online only.

it was used for 10 days. Patients were evaluated regularly and underwent their final assessment an average of 40 months (SD 13.7 months) after surgery. At that time, we used ImageJ 1.49 public-domain software to make measurements using either photos or still images from video footage to determine the return of range of abduction and external rotation. The ranges of abduction and external rotation were measured with the patient standing. External rotation was measured with the patient standing, his/ her shoulder fully internally rotated and forearm placed transversally over the abdomen to avoid overlooking any degree of recovery. For abduction assessment, footage or photographs were recorded from the front, whereas for external rotation assessment images were obtained from above.

\section{Statistical Analysis}

Unpaired Student t-tests were used to compare the mean range of abduction recovery between a) those patients who underwent repair using the classic versus the staged/extended approach; b) between patients with graftable roots and those with total avulsion and paralysis of the serratus anterior muscle; and c) between patients with recovered elbow flexion versus those with persistent paralysis of elbow flexion. We used an unpaired Student t-test to compare age, interval between accident and surgery, and duration of postoperative follow-up between the patients who underwent the classic or the staged/extend approach. We evaluated differences in the number of cases in which patients recovered some degree of external rotation and the number of failures in abduction recovery between the classic and the staged/extended approach using a $2 \times 2$ contingency table.

Statistical significance was set as $\mathrm{p}<0.05$ and all tests were 2-tailed.

\section{Results}

There were no statistically significant differences between groups with respect to patient age at the time of surgical treatment, length of time between injury and surgery, and duration of postoperative follow-up (Table 1).

The overall mean range of abduction recovery $(\mathrm{n}=$ 110) was $58.5^{\circ}\left(\mathrm{SD} 26^{\circ}\right)$. Comparing abduction in patients treated before (2002-2004) and after distal suprascapular nerve exploration (2005-2012), the range of abduction recovery was $45^{\circ}\left(\mathrm{SD} 25.1^{\circ}\right)$ versus $62^{\circ}$ (SD $25.3^{\circ}$ ), respectively, a difference that was statistically significant $(\mathrm{p}=$ 0.002 ).

On average, patients with total avulsion $(n=9)$ recovered $51^{\circ}\left(\mathrm{SD} 22.1^{\circ}\right)$ of abduction versus $59^{\circ}\left(\mathrm{SD} 26.5^{\circ}\right)$ among patients with a graftable root $(n=101)$, a difference that was not statistically significant $(\mathrm{p}=0.3)$. Patients with elbow flexion recovery scoring at least M3 $(n=79,73 \%)$ recovered a mean range of abduction of $61^{\circ}\left(\mathrm{SD} 26.9^{\circ}\right)$, while those with persistent elbow flexion palsy $(\mathrm{n}=31$, $28 \%$ ) had a mean range of abduction recovery of $52^{\circ}$ (SD $23.1^{\circ}$ ), a difference that again failed to achieve statistical significance $(\mathrm{p}=0.1)$. However, if we considered only patients who recovered $30^{\circ}$ of abduction or more (i.e., successful cases), the mean average abduction in patients with versus without recovery of elbow flexion was $66^{\circ}$ (SD $\left.23.2^{\circ}\right)$ and $53.5^{\circ}\left(\mathrm{SD} 21.5^{\circ}\right)(\mathrm{p}=0.01)$, respectively.

Failure of recovery of less than $30^{\circ}$ of abduction was observed in $10(9 \%)$ of the 110 patients. Failure was not observed in any of the 9 patients with total avulsion. We identified a failure rate of $25 \%$ among patients operated on from 2002 through 2004, whereas this rate dropped to $5 \%$ after the introduction of the staged/extended approach (2005-2012). Differences in the failure rate were significant $(\mathrm{p}=0.01)$. Among the 4 cases of failure between 2005 and 2012, in 3 we observed an extended, continuous lesion of the suprascapular nerve up to the level of the suprascapular fossa.

Before the staged/extended approach, 2 of 29 patients recovered active external rotation, one achieving $20^{\circ}$ of external rotation and the other $120^{\circ}$. After adopting the new staged/extended approach, 32 of 81 patients recovered some degree of active external rotation, averaging $87^{\circ}$ (SD $40.6^{\circ}$ ) when measured from full internal rotation (with the patient's forearm starting from resting on his or her abdomen). The number of patients with some recovery of external rotation was significantly higher in the staged/ extended approach group $(\mathrm{p}=0.009)$. In a few of these patients, external rotation was performed with difficulty because of weakness.

Among the 17 patients who required the extended surgical approach, a double lesion of the suprascapular nerve was identified in only 1 patient, who had acromioclavicular dislocation. In this instance, the C-5 and C-6 roots were injured, and the suprascapular nerve also was damaged at the suprascapular notch. Between the root and the scapular notch, the suprascapular nerve was normal in appearance. In this patient, the spinal accessory nerve was dissected up to the level of the medial angle of the scapula and transferred to the suprascapular nerve distal to the scapular notch, without interposition of a nerve graft. In another patient, we identified an extended lesion, 
from the roots of the brachial plexus to the distal third of the suprascapular nerve, but the suprascapular nerve was not affected at the suprascapular notch.

Dissection of the spinal accessory nerve within the deep cervical fascia was straightforward, and, when needed, detaching the trapezius muscle from the clavicle and acromion ensured a very long nerve stump with direct coaptation to the suprascapular nerve in all cases (Fig. 2A and $\mathrm{B})$.

Postoperatively, upper trapezius strength was preserved in all patients.

\section{Discussion}

We observed significant differences in the range of abduction recovery between groups. We believe improvements in the stage/extended approach might be 2-fold: a) less dissection and consequently less devascularization of the accessory nerve, and b) detection of extended lesions of the suprascapular nerve ensuring accessory nerve coaptation with a healthy suprascapular nerve distal stump. In a patient with shoulder palsy, shoulder elevation (i.e., shrugging) via activation of the trapezius is a natural instinct when trying to raise one's arm. This renders spinal accessory to suprascapular nerve transfers synergistic and explains the rapid, easy learning of transfer activation that we observed. Our patients not only appreciated their recovery of abduction, but also the correction of shoulder subluxation thanks to reinnervation of the supraspinatus muscle. They also appreciated the ability to control their shoulder, which they believed improved their balance while walking. Note that each arm swings in synchrony with its contralateral leg. ${ }^{17}$ Swinging the arms enhances gait stability and decreases energy consumption..$^{13}$ Arm swing is mostly a passive movement that is stabilized by active muscle control. ${ }^{13}$ Without muscle control, as in the flail arm observed with brachial plexus lesions, arm swinging mostly disrupts rather than enhances ambulation. Having a reinnervated supraspinatus muscle and being able to control their arm swings might be the reason our patients reported improved walking postoperatively.

In patients who recovered at least $30^{\circ}$ of abduction, we observed that concomitant recovery of elbow flexion improved their abduction recovery range significantly, by an average of $13^{\circ}$. In patients with less than $30^{\circ}$ of abduction recovery, successful elbow flexion reconstruction did not influence the final range of abduction. These results suggest that, provided some initial abduction is recovered, activation of the biceps probably accounts for at least some of the increased range of motion that is observed.

In our patients with total avulsion and consequent palsy of the serratus anterior muscle, the range of abduction recovery was essentially the same as in patients with partial function of the serratus anterior due to preservation of its C-5 root-based innervation. Hence, contrary to what has been proposed previously, ${ }^{16}$ functional preservation of the serratus anterior muscle is not a sine qua non for abduction recovery using spinal accessory to suprascapular nerve transfers.

Once we started transferring the spinal accessory to the suprascapular nerve, our overall failure rate, with failure
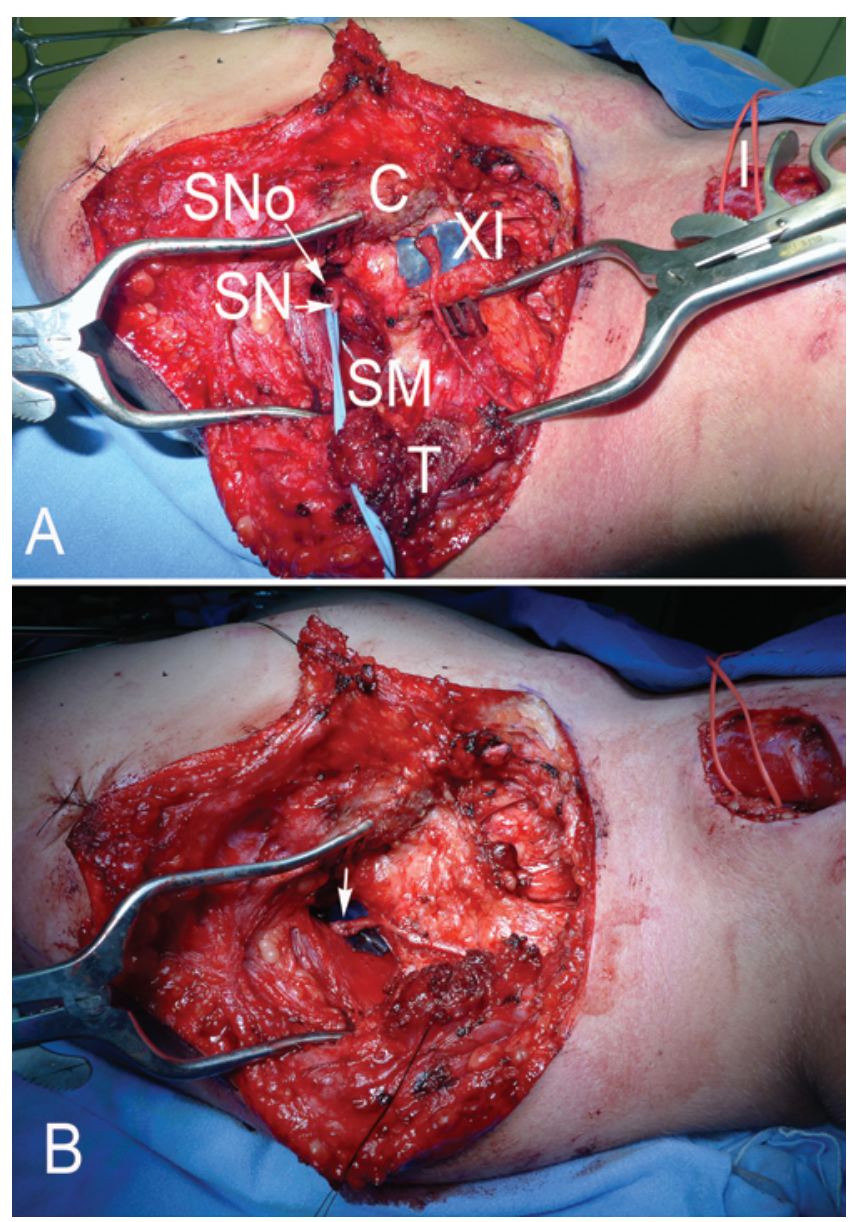

FIG. 2. A: Intraoperative view of the extended anterior approach in the left shoulder and supraclavicular region. The trapezius muscle (T) was detached from the clavicle and acromion, allowing for exposure of the suprascapular fossa and harvesting of a long stump of the spinal accessory nerve $(\mathrm{XI}) . \mathrm{C}=$ clavicle; $\mathrm{SNo}=$ scapular notch; $\mathrm{SN}=$ suprascapular nerve; SM = supraspinatus muscle. B: Intraoperative view of the coaptation site (arrow) between the spinal accessory nerve and the suprascapular nerve within the suprascapular fossa. Figure is available in color online only.

defined as less than $30^{\circ}$ of abduction recovery, was under $10 \%$. We adopted $30^{\circ}$ of abduction as our threshold to distinguish failure and success because at this range shoulder subluxation has been corrected and patients are clearly able to move the glenohumeral joint. This $30^{\circ}$ threshold has already been recommended before by Millesi ${ }^{15}$ and Narakas. ${ }^{16}$ We cannot fully explain why all our patients did not recover more than $30^{\circ}$ of abduction. Double lesions involving the suprascapular nerve are one possibility; however, the prevalence of double lesions was likely low, because we identified only one such case among the 17 patients who ultimately had the entire course of their suprascapular nerve explored. All our patients had had a traction injury to the brachial plexus with possible undetected microlesions throughout the extent of the donor and recipient nerves, which could have hampered nerve recovery in a few of them.

Before the staged/extended approach was available for 
use, just $7 \%$ of our patients with a total palsy recovered some active external rotation, whereas this rate increased to $40 \%$ afterward. We have no clear explanation for this increased rate of external rotation recovery. Eliminating nerve grafts and allowing for nerve coaptation with a healthy distal stump nearer to the infraspinatus muscle might have contributed to the increased recovery of external rotation. However, external rotation recovery remains poor in agreement with previous reports. ${ }^{10,12}$ We propose 2 possible explanations for the absence of external rotation recovery observed in our patients with complete palsy: 1) The number of myelinated fibers in the accessory nerve is insufficient to match that in the suprascapular nerve (1300 vs 3800 , respectively); ${ }^{10,19}$ being the first to be reinnervated, the supraspinatus muscle attracts more regenerating axons that the infraspinatus. 2) The antagonist musclefor instance, the subscapularis muscle-which is needed for humeral head stabilization, remains denervated.

It is very difficult to compare our results against those reported in previous publications, because range of motion results and separation of total and partial palsies are typically not available. One exception is the study by Bhatia et al., ${ }^{9}$ who reported the results of spinal accessory to suprascapular nerve transfers in 49 patients with total brachial plexus palsy. They observed some recovery of abduction in $43(88 \%)$ of their 49 patients, ranging from $30^{\circ}$ to $45^{\circ}$ in 36 patients and from $70^{\circ}$ to $80^{\circ}$ in the remaining 7 .

It has been emphasized that harvesting the spinal accessory nerve for transfer should be done distal to the upper trapezius motor branches, to avoid paralyzing the upper trapezius and creating a dropped shoulder. ${ }^{4}$ However, sectioning the spinal accessory nerve distal to the upper trapezius motor branches does not mitigate denervation of the middle and lower trapezius. Denervation of the middle and lower trapezius by eventually weakening scapular motion contraindicates later trapezius muscle transfer and shoulder arthrodesis. ${ }^{16}$ Recently, however, it was demonstrated that previous harvesting of the spinal accessory nerve did not interfere with range of abduction or external rotation recovery following shoulder arthrodesis. ${ }^{2}$ Chammas et al. ${ }^{11}$ performed shoulder arthrodesis in 27 patients with brachial plexus palsy and identified no differences relating to whether the spinal accessory nerve had previously been transferred or the serratus anterior was paralyzed. They did find differences in the range of recovery of shoulder abduction depending on whether the upper portion of the pectoralis major was functional or paralyzed. Therefore, the need for future shoulder arthrodesis should not be a reason to avoid transferring the spinal accessory to the suprascapular nerve.

We always initiate surgery using the limited approach; but if a healthy suprascapular nerve stump cannot be located, we extend the initial approach by detaching the trapezius from the clavicle. Frequently in patients with total palsy, traction injury to the brachial plexus moves the origin of the suprascapular nerve distally. The suprascapular nerve may lie behind the clavicle and, despite its identification, further dissection may be necessary to achieve proper microsurgical coaptation with the spinal accessory nerve. In more complicated situations, we try to identify the suprascapular nerve directly within the suprascapular fossa. Examples of such instances include when the suprascapular nerve lies inferior to the clavicle and cannot be identified in the cervical region; when scar tissue occupies the retro-clavicular space, as following fractures or dislocations of the clavicle, thereby requiring prior bone repair; and when there is a scapular fracture that accounts for extended lesions of the suprascapular nerve. After the trapezius has been detached, the spinal accessory nerve can be dissected further distally, up to the medial angle of the scapula, which in our series allowed for direct coaptation with the suprascapular nerve in all patients.

We did not consider repairing the suprascapular nerve with any available root stump and reconstructing elbow flexion by transferring the accessory to the musculocutaneous nerve because this strategy requires nerve grafts and consequently leads to poorer results for both transfers. In the past, we have grafted the C-5 root stump to the suprascapular and musculocutaneous nerves to transfer the accessory nerve for triceps or wrist extension reconstruction. The end results were co-contractions between shoulder abduction and elbow flexion. Patients were not able to move the shoulder without flexing the elbow and vice versa (unpublished observations).

Two major limitations of the current paper include the retrospective nature of data collection and the lack of blinded evaluations, because the surgeon who operated on patients also evaluated their shoulder recovery. Although it is possible that his nonblinded evaluations biased results, we believe that such bias is minimal since range of motion was assessed using computer-generated measurements of photographs. We cannot rule out that surgical experience might have contributed to differences in the outcomes in the staged/extended approach group. A significant number of patients (147 of 257) were excluded from the study. Twenty-four patients did not meet our inclusion criteria: preserved accessory nerve function and surgery within the 1st year. Additionally and most importantly, 123 patients were eliminated from the study because the minimum follow-up requirement of 24 months was not met. We have no clear explanation for why these patients did not come back for longer follow-up. We presume that it was because of socioeconomic issues, because of long distances between where they lived and our medical center, or because they were rehabilitated and had gone back to work. Despite this high number of dropouts, a large cohort of 110 patients remained available for the study. We have not provided data about strength recovery because the classic Medical Research Council (MRC) scale is, in our opinion, not appropriate for shoulder evaluation because it does not incorporate range of motion. ${ }^{18}$ Provided that measurements are carried out with the patient standing, abduction range of motion is assessed against gravity. Hence, any degree of active motion recovery requires at least an M3 level of strength. Meanwhile, the strength needed to raise the limb to $30^{\circ}$ against gravity is different from that needed to raise it to $60^{\circ}$, due to the limb's weight. It is reasonable to assume that all our patients with more than $30^{\circ}$ of $\mathrm{ab}$ duction recovery (i.e., 83 of our 110 patients) would have scored M4, if resistance was applied with the limb at $30^{\circ}$ of abduction, as has been suggested before for the evaluation of abduction strength. ${ }^{3}$ Evaluation of the impact of our 
surgery on patients' quality of life has not been done and should be addressed in the future.

Some may question why we did not perform postoperative electrophysiological studies. However, electromyography findings do not correlate with functional clinical recovery after accessory to suprascapular nerve transfers, as previously demonstrated..$^{2}$

In our hands, spinal accessory to suprascapular nerve transfers produce useful recovery in the large majority of patients.

\section{References}

1. Allieu Y: Evolution of our indications for neurotization. Our concept of functional restoration of the upper limb after brachial plexus injuries. Chir Main 18:165-166, 1999

2. Atlan F, Durand S, Fox M, Levy P, Belkheyar Z, Oberlin C: Functional outcome of glenohumeral fusion in brachial plexus palsy: a report of 54 cases. J Hand Surg Am 37:683-688, 2012

3. Bertelli JA, Ghizoni MF: Abduction in internal rotation: a test for the diagnosis of axillary nerve palsy. J Hand Surg Am 36:2017-2023, 2011

4. Bertelli JA, Ghizoni MF: Combined injury of the accessory nerve and brachial plexus. Neurosurgery 68:390-396, 2011

5. Bertelli JA, Ghizoni MF: Improved technique for harvesting the accessory nerve for transfer in brachial plexus injuries. Neurosurgery 58 (4 Suppl 2):ONS366-ONS370, 2006

6. Bertelli JA, Ghizoni MF: Nerve root grafting and distal nerve transfers for C5-C6 brachial plexus injuries. J Hand Surg Am 35:769-775, 2010

7. Bertelli JA, Ghizoni MF: Reconstruction of complete palsies of the adult brachial plexus by root grafting using long grafts and nerve transfers to target nerves. J Hand Surg Am 35:1640-1646, 2010

8. Bertelli JA, Ghizoni MF: Transfer of the accessory nerve to the suprascapular nerve in brachial plexus reconstruction. J Hand Surg Am 32:989-998, 2007

9. Bhatia A, Shyam AK, Doshi P, Shah V: Nerve reconstruction: A cohort study of 93 cases of global brachial plexus palsy. Indian J Orthop 45:153-160, 2011

10. Bonnel F, Allieu Y, Sugata Y, Rabischong P: Bases anatomochirurgicales des neurotisations pour avulsions radiculaires du plexus brachial. Anat Clin 1:291-296, 1979

11. Chammas M, Goubier JN, Coulet B, Reckendorf GMZ, Picot
MC, Allieu Y: Glenohumeral arthrodesis in upper and total brachial plexus palsy. A comparison of functional results. J Bone Joint Surg Br 86:692-695, 2004

12. Malessy MJA, de Ruiter GCW, de Boer KS, Thomeer RTWM: Evaluation of suprascapular nerve neurotization after nerve graft or transfer in the treatment of brachial plexus traction lesions. J Neurosurg 101:377-389, 2004

13. Meyns P, Bruijn SM, Duysens J: The how and why of arm swing during human walking. Gait Posture 38:555-562, 2013

14. Midha R: Nerve transfers for severe brachial plexus injuries: a review. Neurosurg Focus 16(5):E5, 2004

15. Millesi H: Surgical treatment of post-traumatic brachial plexus lesions. Eur Surg 35:191-195, 2003

16. Narakas AO: Brachial plexus lesions, in Leung PC, Gu YD, Ikuta Y, et al (eds): Microsurgery in Orthopaedic Practice. Singapore: World Scientific, 2012, pp 188-254

17. Park J: Synthesis of natural arm swing motion in human bipedal walking. J Biomech 41:1417-1426, 2008

18. Paternostro-Sluga T, Grim-Stieger M, Posch M, Schuhfried O, Vacariu G, Mittermaier C, et al: Reliability and validity of the Medical Research Council (MRC) scale and a modified scale for testing muscle strength in patients with radial palsy. J Rehabil Med 40:665-671, 2008

19. Vathana T, Larsen M, de Ruiter GCW, Bishop AT, Spinner RJ, Shin AY: An anatomic study of the spinal accessory nerve: extended harvest permits direct nerve transfer to distal plexus targets. Clin Anat 20:899-904, 2007

\section{Disclosures}

The authors report no conflict of interest concerning the materials or methods used in this study or the findings specified in this paper.

\section{Author Contributions}

Conception and design: both authors. Acquisition of data: Bertelli. Analysis and interpretation of data: both authors. Drafting the article: both authors. Critically revising the article: Bertelli. Reviewed submitted version of manuscript: Bertelli. Approved the final version of the manuscript on behalf of both authors: Bertelli. Statistical analysis: Bertelli.

\section{Correspondence}

Jayme A. Bertelli, Rua Newton Ramos 70, apto 901, Florianópolis, Santa Catarina 88015395, Brazil. email: drbertelli@gmail. com. 